\title{
Non-Painful Peripheral Inflammation Blocks Conditioned Place-Preference to Morphine and Nicotine through an Ibuprofen-Sensitive and an Ibuprofen Insensitive Pathway
}

\author{
Marco Antonio Sotomayor-Sobrino', Abraham Ochoa-Aguilar ${ }^{1}$, Eda Patricia Tenorio², \\ Martha León-Olea ${ }^{3}$, Roberto Velasco-González ${ }^{1}$, Israel Luna-Mendoza ${ }^{1}$, \\ Claudia Gómez-Acevedo ${ }^{*}$ \\ ${ }^{1}$ Departmento de Farmacología, Facultad de Medicina, Universidad Nacional Autónoma de México, Ciudad de México, México \\ ${ }^{2}$ Departmento de Bioquímica, Facultad de Medicina, Universidad Nacional Autónoma de México, Ciudad de México, México \\ ${ }^{3}$ Instituto Nacional de Psiquiatría "Ramón de la Fuente Muñiz", Ciudad de México, México \\ Email: ${ }^{*}$ goac@unam.mx
}

How to cite this paper: Sotomayor-Sobrino, M.A., Ochoa-Aguilar, A., Tenorio, E.P., León-Olea, M., Velasco-González, R., LunaMendoza, I. and Gómez-Acevedo, C. (2018) Non-Painful Peripheral Inflammation Blocks Conditioned Place-Preference to Morphine and Nicotine through an Ibuprofen-Sensitive and an Ibuprofen Insensitive Pathway. Journal of Behavioral and Brain Science, $\mathbf{8}$, $57-76$.

https://doi.org/10.4236/jbbs.2018.82004

Received: December 5, 2017

Accepted: February 9, 2018

Published: February 12, 2018

Copyright (c) 2018 by authors and Scientific Research Publishing Inc. This work is licensed under the Creative Commons Attribution International License (CC BY 4.0).

http://creativecommons.org/licenses/by/4.0/ (c) (i) Open Access

\section{Abstract}

The field of neuroimmunology has expanded in recent years providing new insights and therapies into pathologies like stroke, autism, and depression. However, few works explore the relationship between inflammatory stimuli and motivation. Thus, the aim of this study was to determine how non-painful inflammatory stimuli affect reward. To test reward-response, we used the morphine and the nicotine induced conditioned place-preference and placeaversion model in rats with non-painful inflammation. The following inflammatory models were used: non-painful infectious inflammation: $24 \mathrm{hrs}$ prior to conditioning sessions, an injection with Calmette-Guerin bacillus (CGB) $1 \times 10^{7} \mathrm{cfu}$, ip, was administered. Non-painful non-infectious inflammation: 24 hrs prior to conditioning sessions, rats' sciatic nerve was blocked and cut, followed by the injection of carrageenan $(750 \mu \mathrm{l})$ in the paw. We then measured the cytokine concentration to determine the inflammatory profile of each of our models. Finally, we administered ibuprofen to determine if it could prevent the effect of inflammation over conditioned place-preference. We show that carrageenan significantly reduced the morphine-induced reward. Non-painful inflammatory stimulus, CGB and denervation + carrageenan, inhibit the conditioned place-preference to morphine and nicotine, CGB also block conditioned place-aversion to nicotine; carrageenan has no effect on $\mathrm{CPA}$. The administration of ibuprofen reinstates conditioned place-preference 
to morphine and nicotine in the carrageenan model, but has no effect in the CGB model; finally ibuprofen has no effect on CPA. Our data suggest that non-painful-inflammatory stimuli inhibit the reward system, independent of cytokine concentration. Furthermore, the administration of a PGE 2 inhibitor can importantly modulate this phenomenon.

\section{Keywords}

Reward, Addiction, Pain, Inflammation, Prostaglandins, Interferon

\section{Introduction}

The brain motivation system is an intricate set of interconnected structures with powerful effects on behavior, eliciting a range of conducts from normal and necessary to pathological. The influence of this system is particularly relevant within the context of substance addiction [1]. Studies in animal models have shown that repeated exposure to a drug promotes robust self-administration, conditioned place-preference and consumption preference [1], while in humans, it generates compulsive use and dependence [2]. These effects are the culprit of the current drug-use epidemics such as tobacco and opioids consumption in the world [2] [3]. The aforementioned substances act through the reward system; once they reach the brain they bind to their receptors, $\mathrm{N}_{2}$ nicotinic or Mu opioid respectively, in the ventral tegmental area (VTA) increasing dopamine and glutamate release towards various structures in the motivation system such as the prefrontal cortex (PFC) and the nucleus accumbens (NAc) [4] [5], causing conditioned responses and reinstatement to the drugs [4]. Rats self-administrate $\mathrm{Mu}$ opioid agonists directly into the VTA, since in conditioned place-preference (CPP) models Mu opioid agonism facilitates CPP, whereas its antagonism blocks these responses [6]. Moreover, morphine administration has also been reported to activate dopaminergic neurons indirectly through an interneuron pathway [7] and the administration of dopamine antagonists in the NAc has shown to diminish the opioid self-administration response [8]; however, the role of dopamine in opioid reward and addiction is inconsistent and, hence, it is often not considered as the key mechanism [9].

Nicotine, on the other hand, has a strong dependence on dopamine neurotransmission for its conditioning effects; upon receptor activation, nicotine increases dopamine and glutamate release to the NAc [10].

Nicotine has reinforcing effects on intravenous self-administration while CPP models have a positive subjective response in drug discrimination that causes motor sensitization, all hallmarks of dopaminergic activity on the reward system [11] [12]. There is currently no effective treatment for drug compulsion and addiction; however, we believe one could be developed from the neural-immune interactions. Recently, the field of neuro-immunology has offered new insights about the role of inflammatory mediator as modulators of behavior and brain 
function. In animal models of allodynia and hyperalgesia, chemokine production can alter opioid receptor responses measured as an increased response to painful stimuli, and a decrease in electrophysiological response when chemokines are released [13] [14]. Several studies in humans have shown that patients with psychiatric diseases such as depression depict an inflammatory status as they present higher concentrations of $\mathrm{C}$ reactive protein in plasma. Accordingly, patients who receive long-term treatments with proinflammatory cytokines present alterations in motivation and motor function [15] [16]. Additionally, animal models have shown that inflammation can impair the dopaminergic system; for example, carrageenan administration in rats decreases dopamine release, its metabolites concentration and its receptor expression in the insular cortex, an important structure for pain and reward processing [15], while IFN $\alpha$ administration in rhesus monkeys during four weeks generates an anxiety like behavior and a decrease in exploration along with a decrease in dopamine metabolites [16] [17]. Further analysis using addiction models has shown that the chemokine receptors CXCR4 and CX3CR1 are co-expressed with MOR in both neurons and microglial cells at important pain and reward processing structures such as the periaqueductal grey area (PAG), nucleus accumbens and ventral tegmental area [13]. Moreover, the functional interaction between these receptors has been demonstrated in cultivated tissue, where co-administration of CXCL12, CXCR4 ligand, blocks the hyperpolarizing current induced by MOR agonists in the PAG, suggesting that chemokine secretion could lead to opioid receptor desensitization, decreasing the opioid agonists effects [14]. Another report showed that the plantar pre-administration of formalin with the inflammatory response associated abolished the reward response to morphine in a conditioned place-preference model and decreased dopamine concentration in the $\mathrm{Nac}[18]$.

With these in mind, the objective of the paper was to determine if non-painful peripheral inflammation had an effect on the conditioned place-preference response to morphine and to nicotine; since these two have different molecular mechanisms, the effect of inflammation might suggest a molecular route of interaction or a neurotransmitter system. Later on, we sought to demonstrate if a simple, clinically relevant pharmacological intervention had an effect on our reward model. Finally, we established the cytokine profile in our inflammatory models to seek possible mediators.

\section{Materials and Methods}

Male Wistar rats between 200 and $350 \mathrm{~g}$ and 5 to 8 weeks old were maintained with food and water ad libitum, individually housed, following an inverted $12 \mathrm{~h}$ light/dark cycle (light 18 - $6 \mathrm{am}$ ). Every behavioral test was performed in a room with dim red illumination and dampening external noise during the active phase of the rats' diurnal cycle. All experiments depicted herein were performed in accordance with the National Institutes of Health guide for the care and use of laboratory animals and were approved by the Ethics Committee of the Universi- 
dad Nacional Autónoma de México (UNAM). All the solutions were diluted in $\mathrm{NaCl} 0.9 \%$, this solution was also used for the control groups.

\subsection{Conditioning Place Preference CPP Paradigm (Figure 1)}

The conditioning sessions were performed in a place preference apparatus $(60 \times$ $30 \mathrm{cms}$ ) that consisted of three compartments. Compartment A was white with a mesh floor and compartment B was black with a sand-like texture floor, but both have the same size; compartment $\mathrm{C}$ connected the entrances to compartments $\mathrm{A}$ and $\mathrm{B}$, and was grey with a smooth floor. Conditioned place preference tests were performed as previously reported [18] and consisted of an 8-day schedule with four distinct phases, as follows:

\subsubsection{Habituation Phase}

From day 1 to 3, each animal was placed in compartment $\mathrm{C}$ and was allowed to freely explore all the compartments in the apparatus for 30 minutes.

\subsubsection{Pre-Conditioning Test Phase}

On day 4, animals were placed in compartment $C$ with free access to the other compartments. Exploration behavior was recorded for 900 seconds to determine the basal place-preference.

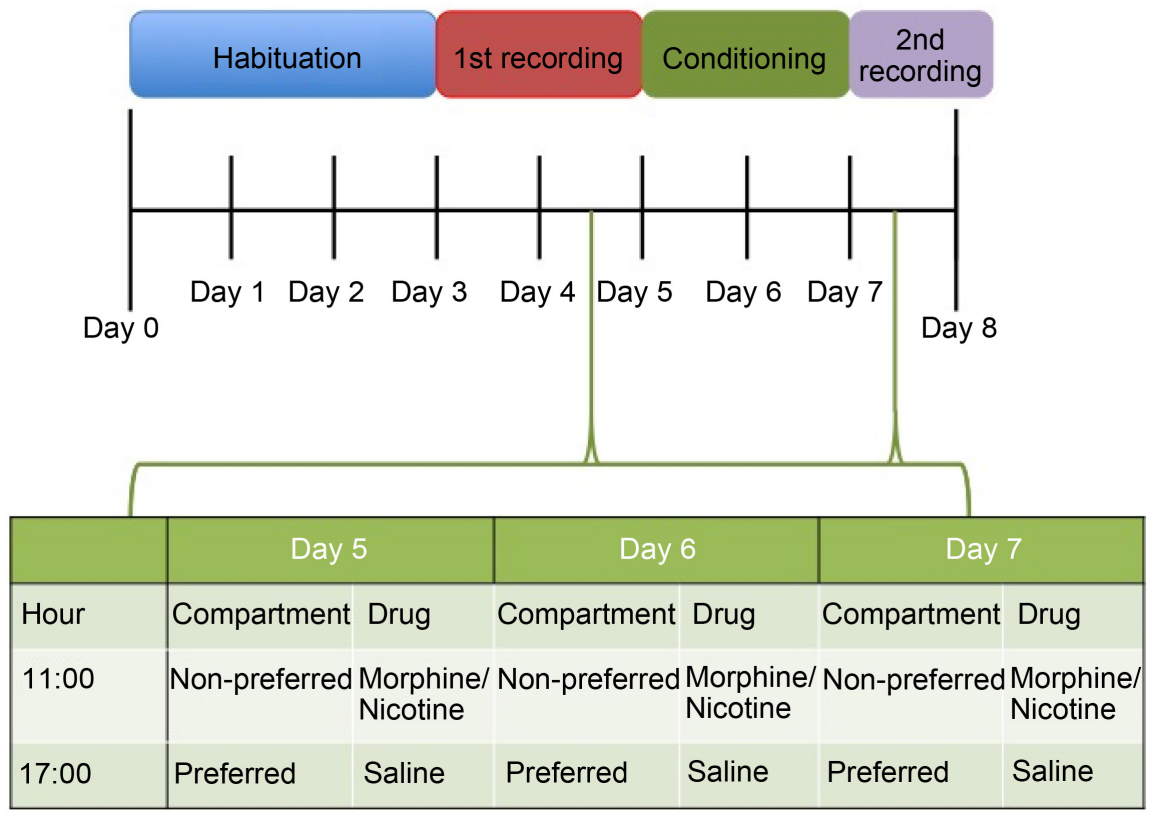

Figure 1. Summary of the experimental design. We used the conditioned place-preference model using a biased procedure to determine the effect of two inflammatory models, carrageenan and CGB administration $24 \mathrm{~h}$ prior to conditioning. Rats were habituated in a three compartment apparatus with a dark, a light and a neutral compartment, we then recorded the animal's conduct in free movement through the apparatus. After $24 \mathrm{~h}$ we began conditioning sessions with saline in the preferred compartment and the drug (morphine/nicotine) in the non-preferred compartment with a $6 \mathrm{~h}$ difference between sessions. On the $8^{\text {th }}$ day the rat was recorded once again to establish the conditioned place-preference. 


\subsubsection{Conditioning Phase}

Conditioning sessions were carried out from day 5 to day 7, when the subjects were exposed to the place preference apparatus for 30minutes. First, animals received a subcutaneous (sc) injection of saline solution. They were locked in the preferred compartment for 30 minutes and returned to their home cage; 6 hours later on the same day, the rewarding drug, morphine $5 \mathrm{mg} / \mathrm{kg}$ or nicotine 1 $\mathrm{mg} / \mathrm{kg}$, (Sigma Aldrich) was administered sc and the animals were again locked in the non-preferred compartment for $30 \mathrm{~min}$ to be returned to their home cage afterwards.

For animals in the nicotine aversion group (nicotine $2 \mathrm{mg} / \mathrm{kg}$ ), the procedure was the same but saline was administered in the non-preferred compartment and nicotine was administered in the preferred compartment. In the control group, every animal received saline in both compartments.

\subsubsection{Post-Conditioning Test Phase}

On day 8 , the animal had again free access to the entire place preference apparatus and exploration conduct was recorded for 900 seconds in order to measure the total time spent in each compartment.

Figure 1 shows the experimental procedure for the conditioning sessions.

\subsection{Experimental Groups}

Painful inflammation (Carr $250+$ Mor) $(\mathrm{n}=10)$ : Subjects received an intraplantar injection of $250 \mu \mathrm{l}$ with $1 \%$ carrageenan $24 \mathrm{hrs}$. prior to the first conditioning session.

Only subjects with reddening, persistent increase in paw volume of at least $50 \%$, audible vocalization and defense upon manipulation of the injected paw, were selected for testing.

Non-painful infectious inflammation (CGB + Mor) $(n=10)$ : Subjects were inoculated intraperitoneally (ip.) with $1 \times 10^{7} \mathrm{CFU}$ of the Calmette-Guerin's bacillus (CGB), Danish strain, 24 hours prior to conditioning.

Only subjects with a loss of at least $5 \%$ of total body weight but who did not present persistent guarding behavior, irritability, vocalization upon abdominal manipulation or any other data of abdominal pain were selected for testing.

Non-painful non-infectious inflammation (Den + Carr 250/750 + Mor) $(\mathrm{n}=$ 30): Subjects were submitted to right sciatic denervation, after which an intraplantar injection of $1 \%$ carrageenan was administered in the concurrent paw (250 or $750 \mu \mathrm{l}$ respectively), and left to rest for $24 \mathrm{hrs}$ before conditioning.

Only subjects with at least $5 \%$ of total body weight loss, reddening and persistent increase in paw volume of at least $50 \%$, but without defense, vocalization upon manipulation of the inflamed paw or any evidence of self-injury behavior suggestive of neuropathic pain were selected for testing.

\subsection{Surgical Technique}

Subjects from the non-painful, non-infectious inflammation group (Den + Carr 
$250 / 750+$ Mor) underwent deafferentation of the sciatic nerve after mild sedation with penthobarbital $(30 \mathrm{mg} / \mathrm{kg}$ ip) as previously reported [7] with some modifications. Briefly, Clorhexidine was applied topically over the area of foray of the right hind leg, and a needle was introduced posteromedially to the greater trochanter pointed in an anteromedial direction. Upon contact with the bone, the needle was withdrawn $1 \mathrm{~mm}$, and bupivacaine $1 \%$ was injected. Bupivacaine was also administered on the skin after 5 minutes of latency. The final volume injected in the deep tissues was $0.5 \mathrm{ml}$ and $0.5 \mathrm{ml}$ on the skin.

The sciatic nerve was then exposed by making a $10 \mathrm{~mm}$ incision over the pelvic joint and deepened through the muscle fascia between the vastuslateralis muscle of the quadriceps and the long head of the biceps femoris. The fibers of the latter were pulled and separated in order to expose the sciatic nerve without damaging its integrity. Upon exposure, $0.3 \mathrm{ml}$ of $1 \%$ bupivacaine was applied topically over the nerve. The efficacy of the block was tested by measuring paw withdrawal latency and vocalization in response to compression in the concurrent paw using a clamp for five minutes after the topical application. Failure to remove the hind paw or to vocalize was regarded as intense nocifensive blockade.

After intense nocifensive blockade was achieved, a $2 \mathrm{~mm}$ segment of the nerve was removed just proximal to its bifurcation into the tibial and common peroneal nerves. The incision was then closed with nylon sutures, followed by the aforementioned intraplantar carrageenan injection.

\subsection{Pharmacological Modulation}

The effect of pharmacological inhibition of the inflammatory process was evaluated by ibuprofen addition. For this test, an ip injection of ibuprofen $50 \mathrm{mg} / \mathrm{kg}$ was administered $15 \mathrm{~min}$ prior to each conditioning session and after the non-painful inflammatory stimulus was induced, rats had free access to 0.6 $\mathrm{mg} / \mathrm{ml}$ ibuprofen in drinking water.

\subsection{Cytokine Measurement}

Serum cytokine concentration was assessed $48 \mathrm{hrs}$ after administration of the inflammatory stimuli (carrageenan/CGB). For blood collection, the animal received a $150 \mathrm{mg} / \mathrm{kg}$ dose of pentobarbital; once unconscious. A midline abdominal incision was made and the intestines were pulled aside exposing the abdominal aorta. The aorta was dissected; blood was collected with a $10 \mathrm{cc}$ syringe and placed in a serum separator tube. Samples were centrifuged for 15 minutes at $2000 \mathrm{~g}$; serum was collected and stored at $-20^{\circ} \mathrm{C}$ until analyzed.

Cytokine concentration was determined by flow cytometry using the CBA, the LEGEND plex ${ }^{\mathrm{Tm}}$ Rat inflammation Panel (Biolegend, San Diego CA) following the instructions provided by the manufacturer. Samples were acquired in a FACScan cytometer (Becton Dickinson, San Jose, CA) at the Laboratorio Nacional de Citometría, IIB, UNAM and analyzed using the FlowJo Software V. 10. (FlowJo LLC, Ashland OR). 


\subsection{Data Analysis}

The variation in time spent in the non-preferred compartment due to morphine conditioning (CPP) was calculated by subtracting the time spent at the non-preferred compartment during pre-conditioning to the time spent at the non-preferred compartment after conditioning for each experimental group.

$\mathrm{CPP}=$ Total time spent in non-preferred compartment after conditioning Total time spent in non-preferred compartment pre-conditioning.

The normality of data distribution was calculated using the D'Agostino \& Pearson omnibus normality test.

Statistical differences between groups were evaluated through a one-way analysis of variance (ANOVA) followed by a Dunnett or Tukey test using the PRISM software (GraphPad); $\mathrm{p}<0.05$ was considered as significant.

To determine effect size, we calculated the difference of means in the experimental groups.

In Table 1 we present a summary of population, conditioned place-preference score and confidence interval per group.

Table 1. Summary of the n, mean CPP or CPA and confidence interval of each of the experimental groups.

\begin{tabular}{|c|c|c|c|}
\hline Group & $\mathbf{n}$ & Mean CPP & $95 \% \mathrm{CI}$ \\
\hline Control & 10 & -3.5 & $-79.1,72.2$ \\
\hline Morphine & 10 & 180.5 & $127.6,233.3$ \\
\hline Carr250 + Mor & 9 & 63.6 & $-25.8,153$ \\
\hline Carr250 + Den + Mor & 9 & 190 & $39.6,340.3$ \\
\hline Carr750 + Den + Mor & 9 & 11.6 & $-76.7,99.9$ \\
\hline CGB + Mor & 10 & 34.1 & $-40.4,108.6$ \\
\hline Mor + Ibu & 8 & 111.9 & $31.7,192.2$ \\
\hline Carr750 + Den + Mor + Ibu & 8 & 487.9 & $399.1,576.6$ \\
\hline $\mathrm{CGB}+\mathrm{Mor}+\mathrm{Ibu}$ & 8 & -150.7 & $-238.9,-62.5$ \\
\hline Nicotine $1 \mathrm{mg} / \mathrm{kg}$ & 12 & 136.6 & $57.1,215.1$ \\
\hline Carr750 + Den + Nic 1 mg/kg & 12 & -6.1 & $-66.1,53.8$ \\
\hline $\mathrm{CGB}+\mathrm{Nic} 1 \mathrm{mg} / \mathrm{kg}$ & 12 & 6.6 & $-52.7,65.9$ \\
\hline Carr750 + Den + Nic 1 mg/kg + Ibu & 10 & 88.7 & $7.2,170.2$ \\
\hline $\mathrm{CGB}+\mathrm{Nic} 1 \mathrm{mg} / \mathrm{kg}+\mathrm{Ibu}$ & 10 & -16.2 & $-65.7,33.4$ \\
\hline Nicotine $2 \mathrm{mg} / \mathrm{kg}$ & 12 & -129.1 & $-139.9,-118.3$ \\
\hline Carr750 + Den + Nic 2 mg/kg & 12 & -182.2 & $-270.4,-93.9$ \\
\hline $\mathrm{CGB}+\mathrm{Nic} 2 \mathrm{mg} / \mathrm{kg}$ & 12 & 35.2 & $-63.7,134.1$ \\
\hline Carr750 + Den + Nic 2 mg/kg + Ibu & 10 & -115.9 & $-306.8,75.1$ \\
\hline $\mathrm{CGB}+\mathrm{Nic} 2 \mathrm{mg} / \mathrm{kg}+\mathrm{Ibu}$ & 10 & -63.9 & $-98.2,-29.5$ \\
\hline
\end{tabular}




\section{Results}

\subsection{Inflammation Inhibits Morphine Induced Place-Preference}

In order to determine if inflammation can influence morphine induced CPP, we administered carrageenan in the right hind paw of rats 24 hours previous to conditioning sessions. The following three days, animals received morphine in the non-preferred compartment and saline in the preferred compartment and in the $4^{\text {th }}$ day, we tested place-preference again.

Figure 2 shows that morphine increased CPP (180.5) compared to that of saline (-3.5), an effect that decreased significantly when rats were previously treated with carrageenan (63.5), showing that in the context of painful inflammation, rats are less prone to show reward to morphine. Although these results agree with previous reports [18], this model does not allow us to determine if $\mathrm{CPP}$ reduction is a consequence of the inflammatory mediators or of the pain induced.

Thus, in order to evaluate the role of inflammatory mediators only, we eliminated pain in our system by performing a deafferentation of the right hind paw before we evaluated the effect of carrageenan administration in CPP. Figure 3 shows that injection of $250 \mu \mathrm{l}$ carrageenan $1 \%$ had no effect on CPP, but that a higher dose of $750 \mu \mathrm{l}$ carrageenan $1 \%$ reduced CPP in 93\% (180.5 vs $11.6 \mathrm{p}<$ 0.0005). Our results show that non-painful inflammation induced with a high concentration of carrageenan blocks CPP. However, the lack of response observed with the lower carrageenan dose suggests that pain can also facilitate this process, so we decided to use a second model to induce painless inflammation to further determine this phenomenon.

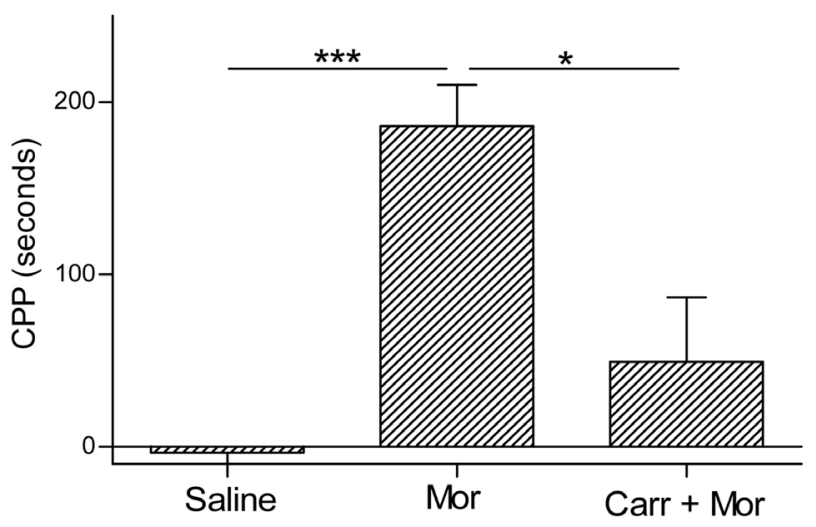

Figure 2. Painful inflammation with carrageenan inhibits morphine-induced place-preference. Rats conditioned with morphine had a significant conditioned place-preference. Rats with carrageenan-induced painful inflammation showed a significant reduction in reward response; this CPP had no significant differences with the saline group. This was an important validation of our model since it replicated the effect previously found by Narita; Kishimoto et al. [18]. ANOVA post hoc Dunnett control was the morphine group. Each bar represents the mean with SEM for 9 to 10 rats. ${ }^{*} \mathrm{p}<0.05,{ }^{* *} \mathrm{p}<0.001$. 


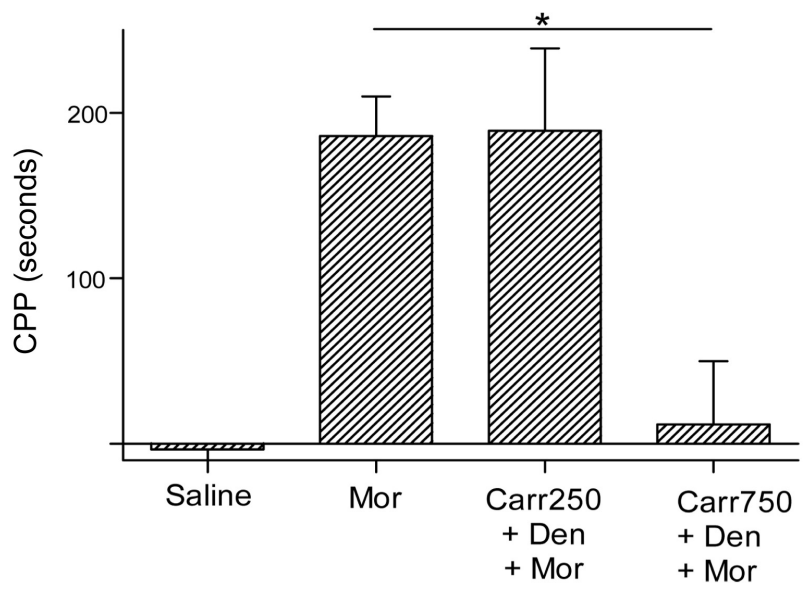

Figure 3. Non-painful inflammation with carrageenan inhibit morphine-induced place-preference. In rats with deafferentation of the right hind paw the low dose carrageenan administration had no effect on morphine-induced place preference, upon increasing the dose of carrageenan we found a $93.6 \%$ reduction in reward response. This showed that non-painful inflammation induced by intraplantar carrageenan administration can prevent morphine-induced place-preference when given in a higher dose. When given a lower dose only accompanied by pain can it prevent placepreference, suggesting a synergy between these processes in their anhedonic effect. ANOVA post hoc Dunnett control was the morphine group. Each bar represents the mean with SEM for 9 to 10 rats. ${ }^{*} \mathrm{p}<0.05$.

We administered intraperitoneal CGB $24 \mathrm{~h}$ before conditioning and evaluated $\mathrm{CPP}$ as in previous experiments; Figure 4 shows a significant reduction in CPP of $97.9 \%$ when compared to that of control (Mean 180.5 vs. 3.9 respectively, $\mathrm{p}<$ 0.005). These results demonstrate, using two independent models, that inflammation, independently of pain, is enough to inhibit morphine induced CPP, suggesting a role of inflammatory molecules as mediators of the morphine reward system.

\subsection{Inflammation Inhibits Nicotine Induced CPP}

We then evaluated if the reduced reward promoted by non-painful inflammation could be observed with a different substance; nicotine was chosen for this experiment since its conditioning and reward system is far more dependent on dopamine neurotransmission than that of the opioidergic.

In accordance with our previous data, Figure 5 shows that carrageenan administration significantly inhibited nicotine induced CPP (136.6 vs $-19.3 \mathrm{p}<$ 0.005). CGB inflammation also inhibited nicotine induced CPP (136.6 vs $6.6 \mathrm{p}<$ $0.005)$.

These results suggest that the mechanism used by inflammatory mediators to inhibit addiction is independent of the neurotransmitter system key to each 


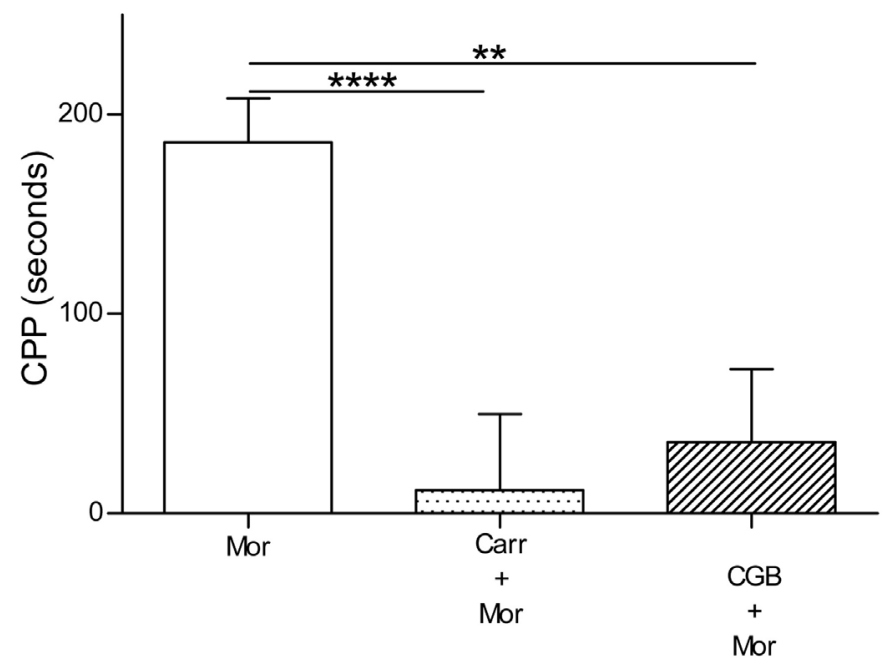

Figure 4. Non-painful inflammation with CGB inhibits morphine-induced place-preference. Animals pretreated with $1 \times 10^{7}$ CFU of Calmette-Guerin bacillus (CGB) exhibited an $81.1 \%$ reduction morphine-induced place-preference. With these our two inflammatory models prevent place-preference. ANOVA post hoc Dunnett control was the morphine group. Each bar represents the mean with SEM for 9 to 10 rats. ${ }^{* *} \mathrm{p}<0.05,{ }^{* * * *} \mathrm{p}<0.001$.

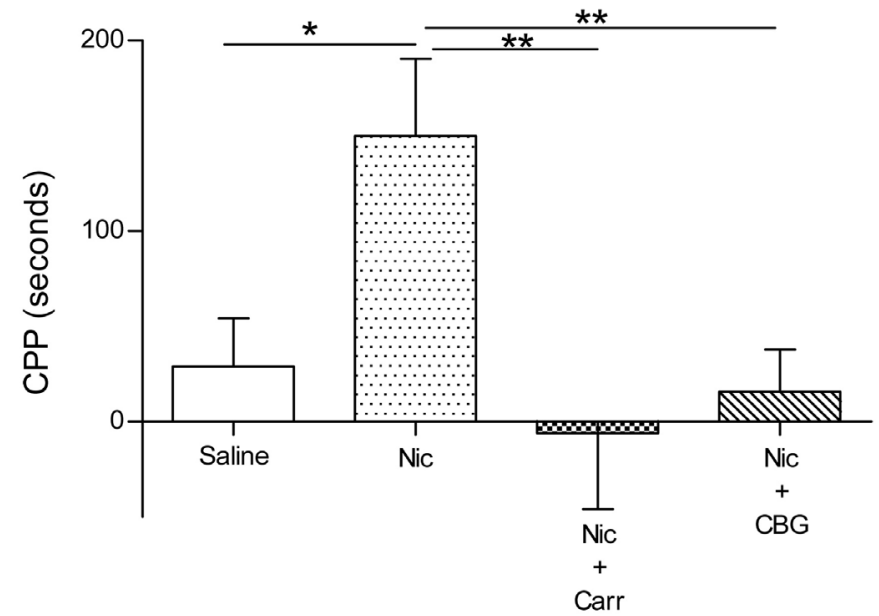

Figure 5. Non-painful inflammation with carrageenan and CGB inhibit nicotine-induced place preference (nicotine $1 \mathrm{mg} / \mathrm{kg}$ ). Nicotine caused a significant conditioned place-preference (1 $\mathrm{mg} / \mathrm{kg}$ ) in rats. ANOVA post hoc Dunnett control was the nicotine group. Each bar represents the mean with SEM for 8 to 10 rats. ${ }^{*} \mathrm{p}<0.05,{ }^{* *} \mathrm{p}<0.001$.

substance.

\subsection{Nicotine-Induced Place Aversion Is Blocked by CGB Non-Painful Inflammation}

Given the clear role of inflammation has on CPP mediators, we aimed to determine if it also mediated CPA using a high nicotine concentration. Figure 6 


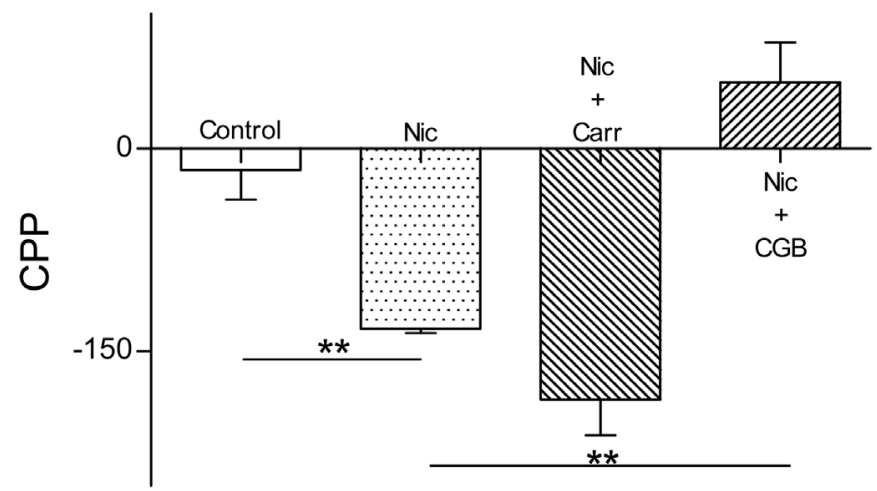

Figure 6. Non-painful inflammation with carrageenan has no effect on nicotine-induced place aversion (nicotine $2 \mathrm{mg} / \mathrm{kg}$ ), CGB blocks it. Nicotine caused a significant conditioned place-aversion (2 $\mathrm{mg} / \mathrm{kg}$ ) in rats, carrageenan had no effect on nicotine-induced place-aversion. CGB on the contrary blocked CPA, ANOVA post hoc Dunnett control was the nicotine group. Each bar represents the mean with SEM for 8 to 10 rats. ${ }^{* *} \mathrm{p}<0.05$.

shows that carrageenan had no significant effect on conditioned place aversion $(-129.1$ vs $-182.2 \mathrm{p}>0.05)$. On the other hand, CGB inhibited nicotine CPA $(-129.1$ vs $35.2 \mathrm{p}<0.05)$. These results show that while carrageenan-induced inflammation has no effect on aversion, CGB infection can inhibit the aversion process.

\subsection{Carrageenan Reduces Serum TNF $\alpha$, IL12 and INF $\gamma$}

We then determined the level of the most commonly evaluated cytokines present in plasma of the rats treated with carrageenan and CGB to characterize usual changes in our models.

So we measured the plasma concentration of TNF $\alpha$, IL12, INF $\gamma$ and IL10.

Carrageenan administration was associated with a significant reduction in $\operatorname{TNF} \alpha$, IL12 and INF $\gamma$ concentration in serum; no effect was observed on IL10. On the other hand, CGB had no effect on cytokine concentration in serum after 48 hours (data shown on Supplementary material).

\subsection{Ibuprofen Modulates the Effect of Inflammation on Morphine Induced CPP}

Several studies suggest that molecules related to acute inflammation could be involved in addiction establishment; prostaglandins (PG) are key mediators of this process and they can act both locally and systemically. We therefore, administered rats with ibuprofen (IBU), a specific PG inhibitor, in order to evaluate if in the absence of PG our inflammatory models could still block drug-induced CPP. Figure 7 shows that ibuprofen per se has no influence on morphine induced CPP. However, when administered along with the carrageenan stimuli, the inhibition effect previously observed was not only reversed, it was also increased twice when compared to that of morphine control exposed animals (487 


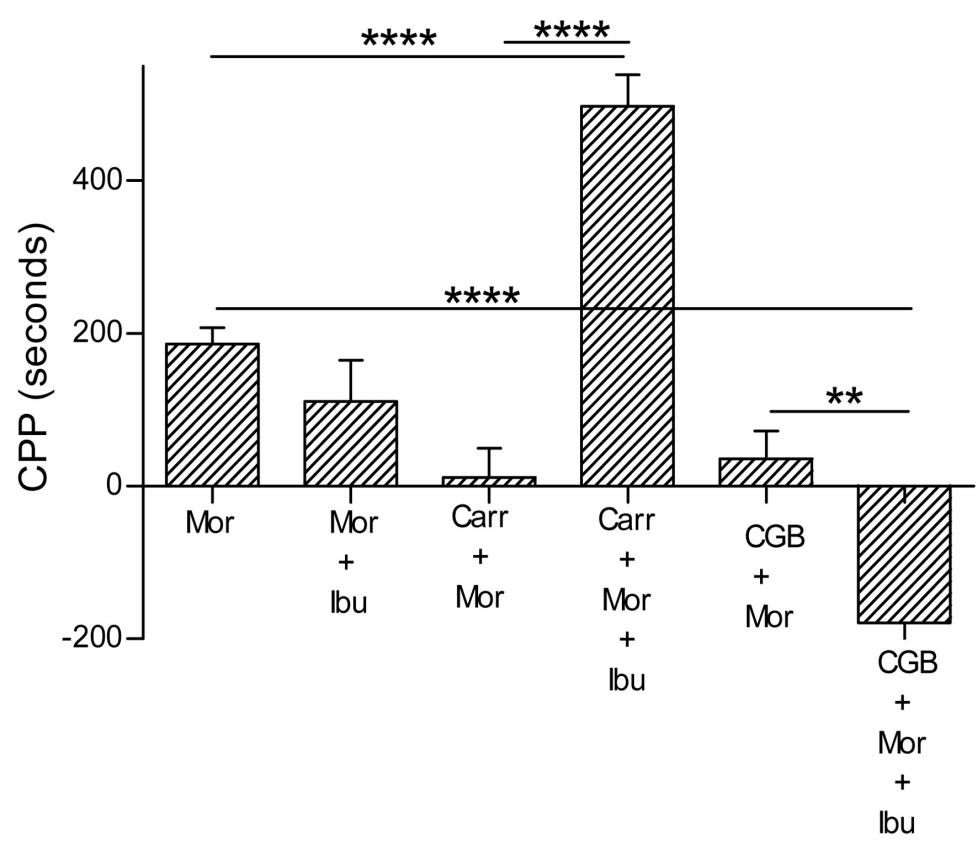

Figure 7. Ibuprofen reinstates morphine induced CPP in the carrageenan model but not in the CGB model. We sought to determine if the administration of ibuprofen, a prostaglandin inhibitor could reinstate morphine-induced place-preference in our carrageenan model, but not in the CGB model. Ibuprofen alone had no pharmacological interactions with morphine in CPP. These results suggest that the two inflammatory models are mechanistically distinct, carrageenan being sensitive to ibuprofen block, while CGB is resistant, suggesting an important role of prostaglandins in the effect found with carrageenan but not of CGB. ANOVA post hoc Dunnett control was the morphine group. Each bar represents the mean with SEM for 8 to 10 rats. ${ }^{* *} \mathrm{p}<0.05,{ }^{* * * *} \mathrm{p}<0.001$.

vs $180.5 \mathrm{p}<0.0004)$. Contrastingly, when IBU was administered using the CGB inflammation model, morphine induced CPP was further inhibited (34.1 vs $-150.7 \mathrm{p}<0.005)$. These results show that prostaglandins can strongly modulate the reward processes since these mediators are a central part of the carrageenan model, while in the CGB model they are absent.

\subsection{Ibuprofen Modulates the Effect of Inflammation on Nicotine Induced CPP and CPA}

We then tested how ibuprofen affected the nicotine-conditioning model.

In Figure 8, we appreciate that IBU only reinstated carr induced CPP $(-19.3$ vs 136), in our CGB inflammatory model ibuprofen had no effect and reward was still blocked (6.6 vs -16.6); thus, following a similar pattern to that observed in CPP with morphine.

In our aversion model Figure 9 shows that ibuprofen has no effect on inflammation induced aversion, meaning that carrageenan could not block aversion with or without IBU ( -182.2 vs -115.9$)$, while CGB blocks aversion despite IBU (35.2 vs 63.9). 


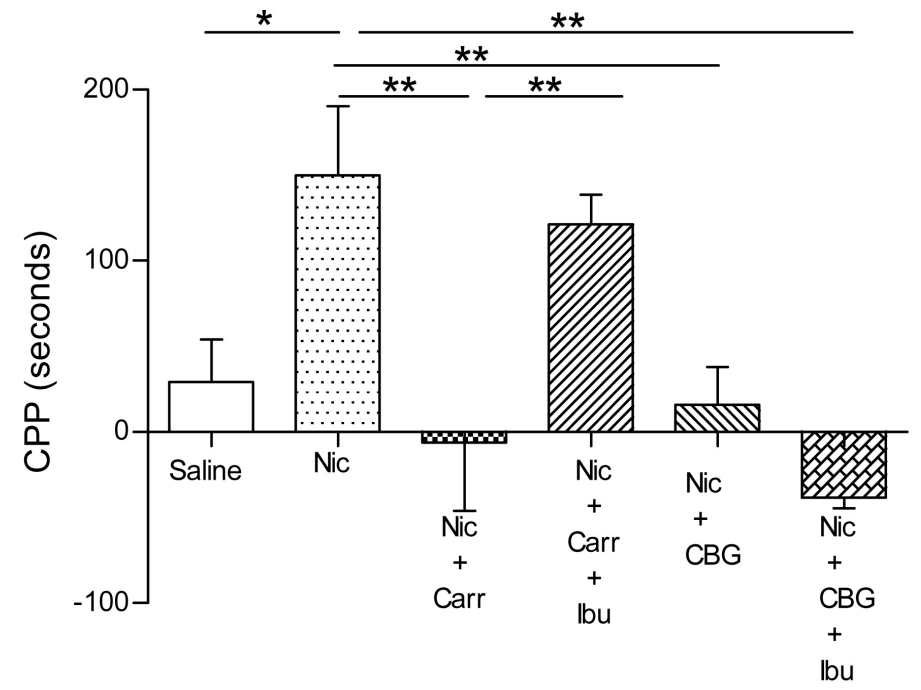

Figure 8. Ibuprofen reinstates nicotine induced CPP in the carrageenan model but not in the CGB model. Administration of ibuprofen reinstated nicotine-induced place-preference in our inflammatory model but not in the CGB model, similar to the results shown for morphine. ANOVA post hoc Dunnett control was the morphine group. Each bar represents the mean with SEM for 8 to 10 rats. ${ }^{*} \mathrm{p}<$ $0.05,{ }^{* *} \mathrm{p}<0.005$.

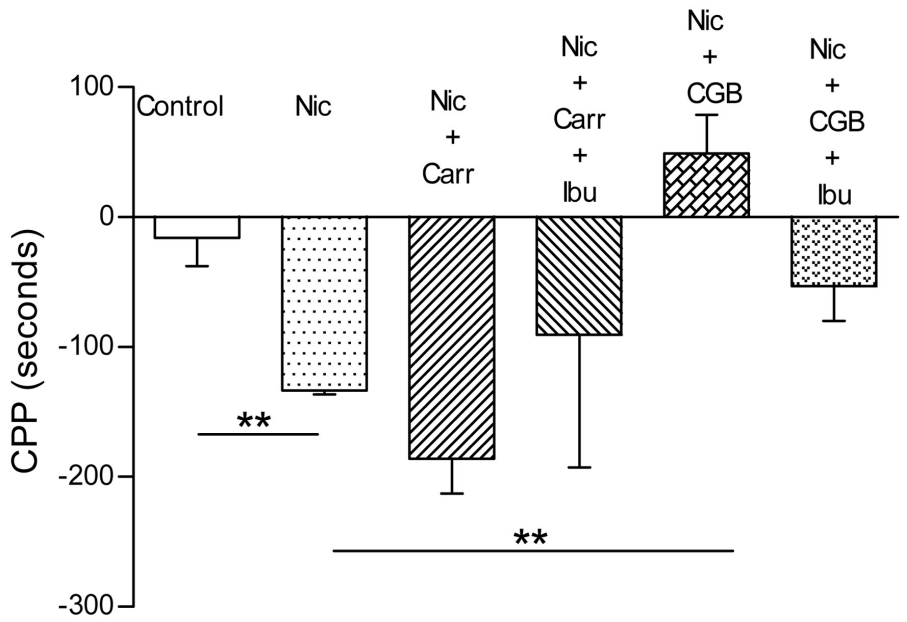

Figure 9. Ibuprofen had no effect on nicotine-induced CPA. Ibuprofen had neither effect in the carrageenan nor the CGB model in nicotine-induced place aversion. ANOVA post hoc Dunnett control was the morphine group. Each bar represents the mean with SEM for 8 to 10 rats. ${ }^{*} \mathrm{p}<0.05,{ }^{* *} \mathrm{p}<0.001$.

\section{Discussion}

Our results support previous findings indicating that painful-inflammation can decrease CPP induced by morphine [18]. However, we also observed an important decrease in CPP and CPA secondary to non-painful inflammatory stimuli.

We started with the carrageenan model because of the previous study demonstrating a significant decrease in morphine induced CPP [18]. However, in the 
work done by Narita and Kishimoto, inflammation was accompanied by pain; thus, to separate these effects in the model hereby presented, we cut the sciatic nerve before administering the carrageenan, assuring that subjects developed similar degrees of inflammation without the painful perception.

We found that the painless low-dose carrageenan group (Carr $250+$ Den + Mor) had a comparable CPP as that of the morphine group, so a low level of inflammation without pain is not sufficient to prevent reward, but with a greater inflammatory stimulus inflammation is once again prevented, suggesting a synergy between pain and inflammation inhibiting the reward induced by opioids.

These results must be considered cautiously since it has been demonstrated that pain itself is a pro-inflammatory stimulus and a nerve blockage can cause a decrease in the inflammatory mediators released by carrageenan injection [19]. Nevertheless, when the dose of carrageenan was increased to $750 \mu$, the CPP also decreased significantly, proving that a non-painful inflammatory stimulus can inhibit the reward response induced by morphine.

Whereas, when used on nicotine-conditioning, a high dose of carrageenan $(750 \mu \mathrm{l})$ could also block the conditioned place-preference, but not the conditioned place-aversion.

This can be explained by the different set of neurotransmitters associated with each conduct, while nicotine, in low doses, increases dopamine and glutamate release to the NAc. Higher doses can increase GABA release from the lateral habenulla ( $\mathrm{LHb})$ to the VTA, which in turn decreases dopamine release and causes aversion [17].

Carrageenan, as mentioned before, can prevent morphine induced CPP. This inhibition is related primarily to an inhibition of the opioidergic system, since DAMGO a $\mu$ opioid agonist can reinstate the preference [18]. However, there are other important effects to be considered after carrageenan administration.

The carrageenan model is characterized by the release of prostaglandins, mainly $\mathrm{PGE}_{2}$ [20]; these mediators have shown an important capability to modulate CNS activity. Tanaka K. showed that $\mathrm{PGE}_{2}$ activity is essential for depressive behavior induced by a chronic social defeat model, as the administration of a prostaglandin antagonist, or a conditional knock-out of prostaglandin receptors, increased the activity of mesocortical dopaminergic pathways in this model and prevented the depressive behavior [21]. Prostaglandin synthesis in the CNS can even induce conditioned place-aversion in other models [22].

These effects could help explain how carrageenan blocks morphine and nicotine CPP, since they can prevent opioid receptor activation[14] and decrease mesocortico-limbic activity and dopamine release [17]; however, they have no effect on nicotine CPA, since this response is mainly mediated by GABA [10] [11].

As a second inflammatory model, we used the Calmette-Guerin bacillus as it has been used in the past to show inflammation-induced depressive-like behavior in the animal model. Mycobacterium administration diminishes $\mathrm{PGE}_{2}$ syn- 
thesis [23] allowing us to work with two well-defined and different models of inflammation.

Upon ip injection of the bacillus, there is a marked increase in IL 12 production by peritoneal macrophages triggering the immune response; eventually, this response gives way to an increase in INF $\gamma$ from T lymphocites [24].

O'Connor and his team demonstrated that the rats that received the ip injection of CGB increased the activity of the tryptophan-degrading enzyme IDO, which in turn led to an increase in brain kynurenine; this increase was dependent on the INF increase [25].

The kynurenine pathway has shown important effects that could potentially modify the reward's system response to certain drugs. Kynurenic acid, for example, is a potent glutamate antagonist, blocking the NMDA and AMPA receptor function, a key step in long-term potentiation in this system [26].

In our work, CGB managed to prevent CPP induced by morphine and by nicotine as well as CPA induced by nicotine. This suggests a common pathway that converges somewhere on the system, a good candidate is dopamine [7] [8] [9] [22] [27].

To determine if our models were susceptible to a pharmacological manipulation, we evaluated a non-selective COX inhibitor, ibuprofen to test its effect on our place conditioning model.

At the doses used in this study, ibuprofen reduces $76 \% \mathrm{PGE}_{2}$ productionin the carrageenan model [28]; this prostaglandin inhibition showed differential effects on our non-painful inflammatory models.

Ibuprofen showed its effect on the carrageenan model, where it consistently reinstated CPP to nicotine and in the morphine group; not only was reward reinstated, but also the CPP was enhanced. This phenomenon, however, was not replicated in morphine-conditioned subjects receiving ibuprofen without inflammation.

This finding strongly suggests that this effect was not induced by a drug-drug interaction between morphine and ibuprofen, but it allows to speculate the existence of an undetermined mediator induced by inflammation but not by pain processes; this mediator was found to alter the rewarding effect of morphine and not to be inhibited by ibuprofen.

CGB treated animals were resistant to modulation by ibuprofen; none of the groups had significant differences upon treatment with the anti-inflammatory drug neither on morphine nor on nicotine conditioning or aversion sessions.

To better understand this immunological profile, we measured cytokine concentration in plasma 48 hours after the induction of the inflammatory models, finding that carrageenan significantly decreases TNF $\alpha$, IL1, IL 12 and INF $\gamma$ at this time set. These results are similar to previous literature in which after 24 hours no significant changes in cytokines are found [29] and even suggests that the higher dose used in our model or the longer follow up ( $48 \mathrm{hrs}$ ) can lead to a decrease in cytokine production [30]. Nevertheless, the relation to conduct can- 
not be explained right now.

Our evidence suggests that there are at least two different and independent peripheral inflammatory phenomena strongly modifying behavioral response in a place-preference model to two drugs with a different neural substrate; one is ibuprofen sensitive while the other is ibuprofen insensitive.

Present findings differ from other studies in the literature. Doctor Larson in 2006 showed that the administration of LPS in rats could diminish sucrose intake, but that it did not affect place-preference induced by sucrose consumption. This could be explained by the different mediators that characterize the immune response to LPS like high levels of IL 1 and TNF, which were absent in our models, and the divergent reward pathways in the brain [31].

However, our results support other studies demonstrating that some chronic inflammatory models can lead to depressive states. For example, the intraperitoneal CGB administration causes a dose dependent depression as increased immobility in a forced swim and tail suspension tests and a decrease in sucrose consumption [22] [32] [33].

Prostaglandins have also an important role in mood disorders; in animal models, prostaglandins are necessary for depression induced by social defeat [21]. Mendlewicz [34] showed that patients with refractory depression and no other comorbidities presented an $80 \%$ remission upon adding low dose aspirin $(160 \mathrm{mg} /$ day) to their antidepressant.

This interaction between inflammatory mediators and the reward system could also lead to the development of more effective treatments for substance abuse and the development of safer chronic pain treatments, as to a better understanding and treatment of depressive disorder.

\section{Acknowledgements}

Marco Antonio Sotomayor Sobrino is a doctoral student from Programa de Doctorado en Ciencias Biomédicas, Universidad Nacional Autónoma de México (UNAM) and received fellowship 514013572 from CONACYT. Authors want to thank doctor Clara Espitia, Head of the Immunology Department from Instituto de Investigación Biomédicas at Facultad de Medicina, UNAM and M.C. Cristina Parada Colín for the generous donation and management of the CGB. Authors also want to thank Mrs. Josefina Bolado, Head of the Scientific Paper Translation Department, from División de Investigación at Facultad de Medicina, UNAM, for editing the English-language version of this manuscript.

This work was supported by grant IA204616 from PAPIIT (DGAPA, UNAM, México).

\section{References}

[1] Lynch, W.J., Nicholson, K.L., Dance, M.E., Morgan, R.W. and Foley, P.L. (2010) Animal Models of Substance Abuse and Addiction: Implications for Science, Animal Welfare, and Society. Comparative Medicine, 60, 177-188.

[2] Alexander, G.C., Frattaroli, S. and Gielen, A.C., Eds. (2015) The Prescription Opio- 
id Epidemic: An Evidence-Based Approach. Johns Hopkins Bloomberg School of Public Health, Baltimore, Maryland.

https://www.jhsph.edu/research/centers-and-institutes/center-for-drug-safety-and-effect iveness/research/prescription-opioids/JHSPH_OPIOID_EPIDEMIC_REPORT.pdf

[3] World Health Organization (2015) WHO Global Report on Trends in Prevalence of Tobacco Smoking 2015. WHO Magazine, 359.

[4] Mercuri, N., Calabresi, P., Stanzione, P. and Bernardi, G. (1985) Electrical Stimulation of Mesencephalic Cell Groups (A9-A10) Produces Monosynaptic Excitatory Potentials in Rat Frontal Cortex. Brain Research, 338, 192-195. https://doi.org/10.1016/0006-8993(85)90267-7

[5] Morales, M. and Margolis, E.B. (2017) Ventral Tegmental Area: Cellular Heterogeneity, Connectivity and Behaviour. Nature Reviews Neuroscience, 18, 73-85.

https://doi.org/10.1038/nrn.2016.165

[6] Badiani, A., Belin, D., Epstein, D., Calu, D. and Shaham, Y. (2011) Opiate versus Psychostimulant Addiction: The Differences Do Matter. Nature Reviews Neuroscience, 12, 685-700. https://doi.org/10.1038/nrn3104

[7] Johnson, S.W. and North, R.A. (1992) Opioids Excite Dopamine Neurons by Hyperpolarization of Local Interneurons. Journal of Neuroscience, 12, 483-488.

[8] Shaham, Y. and Stewart, J. (1996) Effects of Opioid and Dopamine Receptor Antagonists on Relapse Induced by Stress and Re-Exposure to Heroin in Rats. Psychopharmacology, 125, 385-391. https://doi.org/10.1007/BF02246022

[9] Fields, H.L. and Margolis, E.B. (2015) Understanding Opioid Reward. Trends in Neurosciences, 38, 217-225. https://doi.org/10.1016/j.tins.2015.01.002

[10] D’Souza, M.S. and Markou, A. (2011) Neuronal Mechanisms Underlying Development of Nicotine Dependence: Implications for Novel Smoking-Cessation Treatments. Addiction Science \& Clinical Practice, 6, 4-16.

[11] Benowitz, N.L., Hukkanen, J. and Jacob, P. (2009) Nicotine Psychopharmacology. Handbook of Experimental Pharmacology, Vol. 192. Springer-Verlag, Berlin, Heidelberg, 29-60. https://doi.org/10.1007/978-3-540-69248-5_2

[12] Domino, E.F. (2001) Nicotine Induced Behavioral Locomotor Sensitization. Progress in Neuro-Psychopharmacology and Biological Psychiatry, 25, 59-71. https://doi.org/10.1016/S0278-5846(00)00148-2

[13] Kress, M. (2010) Nociceptor Sensitization by Proinflammatory Cytokines and Chemokines. The Open Pain Journal, 3, 97-107. https://doi.org/10.2174/1876386301003010097

[14] Heinisch, S., Palma, J. and Kirby, L.G. (2011) Interactions between Chemokine and $\mathrm{Mu}$-Opioid Receptors: Anatomical Findings and Electrophysiological Studies in the Rat Periaqueductal Grey. Brain, Behavior, and Immunity, 25, 360-372. https://doi.org/10.1016/j.bbi.2010.10.020

[15] Coffeen, U., Ortega-Legaspi, J.M., de Gortari, P., Simón-Arceo, K., Jaimes, O., Amaya, M.I. and Pellicer, F. (2010) Inflammatory Nociception Diminishes Dopamine Release and Increases Dopamine D2 Receptor mRNA in the Rat's Insular Cortex. Molecular Pain, 6, 75. https://doi.org/10.1186/1744-8069-6-75

[16] Felger, J.C., Alagbe, O., Hu, F., Mook, D., Freeman, A.A., Sanchez, M.M. and Miller, A.H. (2007) Effects of Interferon-Alpha on Rhesus Monkeys: A Non-Human Primate Model of Cytokine-Induced Depression. Biological Psychiatry, 62, 1324-1333. https://doi.org/10.1016/j.biopsych.2007.05.026

[17] Felger, J.C. and Miller, A.H. (2012) Cytokine Effects on the Basal Ganglia and Do- 
pamine Function: The Subcortical Source of Inflammatory Malaise. Frontiers in Neuroendocrinology, 33, 315-327. https://doi.org/10.1016/j.yfrne.2012.09.003

[18] Narita, M., Kishimoto, Y., Ise, Y., Yajima, Y., Misawa, K. and Suzuki, T. (2005) Direct Evidence for the Involvement of the Mesolimbic Kappa-Opioid System in the Morphine-Induced Rewarding Effect under an Inflammatory Pain-Like State. Neuropsychopharmacology, 30, 111-118.

[19] Beloeil, H., Gentili, M., Benhamou, D. and Mazoit, J.X. (2009) The Effect of a Peripheral Block on Inflammation-Induced Prostaglandin E2 and Cyclooxygenase Expression in Rats. Anesthesia and Analgesia, 109, 943-950. https://doi.org/10.1213/ane.0b013e3181aff25e

[20] Guay, J., Bateman, K., Gordon, R., Mancini, J. and Riendeau, D. (2004) Carrageenan-Induced Paw Edema in Rat Elicits a Predominant Prostaglandin E2 (PGE2) Response in the Central Nervous System Associated with the Induction of Microsomal PGE2 Synthase-1. Journal of Biological Chemistry, 279, 24866-24872. https://doi.org/10.1074/jbc.M403106200

[21] Tanaka, K., Furuyashiki, T., Kitaoka, S., Senzai, Y., Imoto, Y., Segi-Nishida, E., Narumiya, S., et al. (2012) Prostaglandin E2-Mediated Attenuation of Mesocortical Dopaminergic Pathway Is Critical for Susceptibility to Repeated Social Defeat Stress in Mice. The Journal of Neuroscience: The Official Journal of the Society for Neuroscience, 32, 4319-4329. https://doi.org/10.1523/JNEUROSCI.5952-11.2012

[22] Fritz, M., Klawonn, A.M., Nilsson, A., Singh, A.K., Zajdel, J., BjörkWilhelms, D., Engblom, D., et al. (2016) Prostaglandin-Dependent Modulation of Dopaminergic Neurotransmission Elicits Inflammation-Induced Aversion in Mice. The Journal of Clinical Investigation, 126, 695-705. https://doi.org/10.1172/JCI83844

[23] Yamashita, M., Shinohara, T., Tsuji, S., Myrvik, Q.N., Nishiyama, A., Henriksen, R.A. and Shibata, Y. (2007) Catalytically Inactive Cyclooxygenase 2 and Absence of Prostaglandin E2 Biosynthesis in Murine Peritoneal Macrophages Following in Vivo Phagocytosis of Heat-Killed Mycobacterium bovis Bacillus Calmette-Guérin. Journal of Immunology, 179, 7072-7078. https://doi.org/10.4049/jimmunol.179.10.7072

[24] Van Crevel, R., Ottenhoff, T.H.M. and van der Meer, J.W.M. (2002) Innate Immunity to Mycobacterium tuberculosis. Clinical Microbiology Reviews, 15, 294-309. https://doi.org/10.1128/CMR.15.2.294-309.2002

[25] O’Connor, J.C., Lawson, M.A., André, C., Briley, E.M., Szegedi, S.S., Lestage, J., Kelley, K.W., et al. (2009) Induction of IDO by bacilli Calmette-Guérin Is Responsible for Development of Murine Depressive-Like Behavior. Journal of Immunology, 182, 3202-3212.

[26] Khan, M.J., Seidman, M.D., Quirk, W.S. and Shivapuja, B.G. (2000) Effects of Kynurenic Acid as a Glutamate Receptor Antagonist in the Guinea Pig. European Archives of Oto-Rhino-Laryngology, 257, 177-181. https://doi.org/10.1007/s004050050218

[27] Fowler, C.D. and Kenny, P.J. (2014) Nicotine Aversion: Neurobiological Mechanisms and Relevance to Tobacco Dependence Vulnerability. Neuropharmacology, 76, 533-544. https://doi.org/10.1016/j.neuropharm.2013.09.008

[28] Press, D. (2013) Anti-Inflammatory Agents of the Carbamoylmethyl Ester Class: Synthesis, Characterization and Pharmacological Evaluation. Journal of Inflammation Research, 6, 35-43.

[29] Loram, L.C., et al. (2007) Cytokine Profiles during Carrageenan-Induced Inflammatory Hyperalgesia in Rat Muscle and Hind Paw. The Journal of Pain, 8, 127-136. 
[30] Dooper, M.M.B.W., Wassink, L., M’Rabet, L. and Graus, Y.M.F. (2002) The Modulatory Effects of Prostaglandin-E on Cytokine Production by Human Peripheral Blood Mononuclear Cells Are Independent of the Prostaglandin Subtype. Immunology, 107, 152-159. https://doi.org/10.1046/j.1365-2567.2002.01474.x

[31] Larson, S.J. (2006) Lipopolysaccharide and Interleukin-1 $\alpha$ Decrease Sucrose Intake But Do Not Affect Expression of Place Preference in Rats. Pharmacology Biochemistry and Behavior, 84, 429-435. https://doi.org/10.1016/j.pbb.2006.06.004

[32] Pan, Y., Lin, W., Wang, W., Qi, X., Wang, D. and Tang, M. (2013) The Effects of Central Pro- and Anti-Inflammatory Immune Challenges on Depressive-Like Behavior Induced by Chronic Forced Swim Stress in Rats. Behavioural Brain Research, 247, 323-240. https://doi.org/10.1016/j.bbr.2013.03.031

[33] Moreau, M., André, C., O’Connor, J.C., Dumich, S.A., Woods, J.A., Kelley, K.W., Castanon, N., et al. (2008) Inoculation of Bacillus Calmette-Guerin to Mice Induces an Acute Episode of Sickness Behavior Followed by Chronic Depressive-Like Behavior. Brain, Behavior, and Immunity, 22, 1087-1095.

https://doi.org/10.1016/j.bbi.2008.04.001

[34] Mendlewicz, J., Kriwin, P., Oswald, P., Souery, D., Alboni, S. and Brunello, N. (2006) Shortened Onset of Action of Antidepressants in Major Depression Using Acetylsalicylic Acid Augmentation: A Pilot Open-Label Study. International Clinical Psychopharmacology, 21, 227-231.

https://doi.org/10.1097/00004850-200607000-00005 


\section{Supplementary}
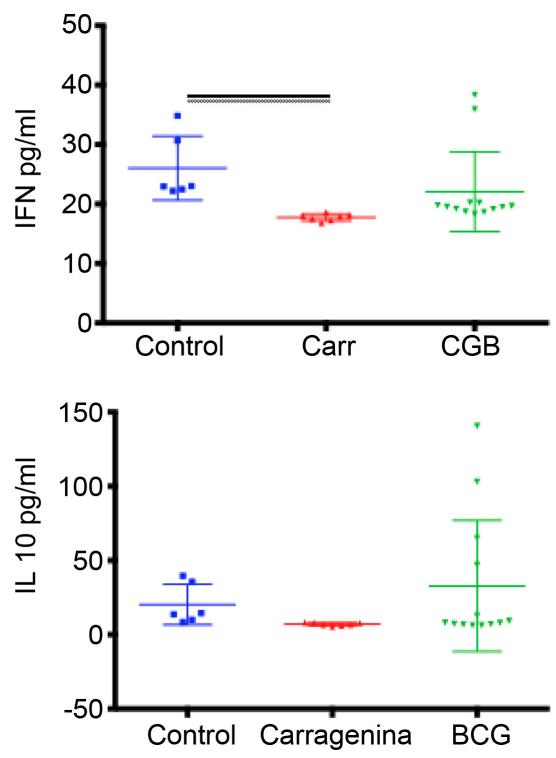
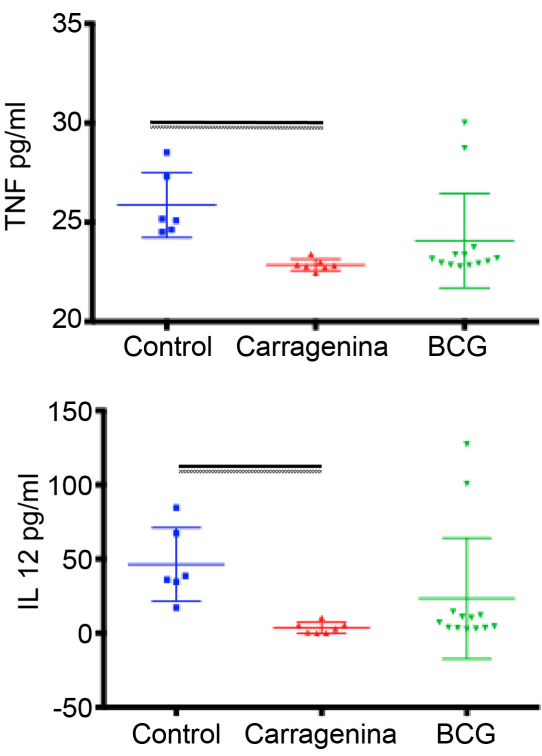

Figure S1. Carrageenan significantly reduces $\operatorname{TNF} \alpha$, IL12 and INF $\gamma$ and has no effect on IL10, CGB has no effect on cytokine concentration. When we measured cytokine concentration in serum of rats in our inflammatory models we found a significant reduction in all of the pro-inflammatory cytokines in the group that received intraplantar carrageenan, there was no significant effect on the anti-inflammatory cytokine IL10, on the other hand CGB has no significative effect on any cytokine measured. ANOVA post hoc Tukey control was the nicotine group, for differences between the inflammatory model and the rats receiving ibuprofen we used a t-test. Each bar represents the mean with SEM for 8 to 10 rats. $\mathrm{p}<0.05$. 